\title{
LOWER BOUNDS FOR ESTRADA INDEX
}

\section{Ivan Gutman}

\author{
Communicated by Slobodan Simić
}

\begin{abstract}
If $G$ is an $(n, m)$-graph whose spectrum consists of the numbers $\lambda_{1}, \lambda_{2}, \ldots, \lambda_{n}$, then its Estrada index is $\operatorname{EE}(G)=\sum_{i=1}^{n} e^{\lambda_{i}}$. We establish lower bounds for $\operatorname{EE}(G)$ in terms of $n$ and $m$.
\end{abstract}

\section{Introduction}

In this paper we are concerned with simple graphs, that have no loops and no multiple or directed edges. Let $G$ be such a graph, and let $n$ and $m$ be the number of its vertices and edges. Then we say that $G$ is an $(n, m)$-graph.

The spectrum of $G$ is the spectrum of its adjacency matrix [1], and consists of the (real) numbers $\lambda_{1}, \lambda_{2}, \ldots, \lambda_{n}$. The number $n_{0}$ of zeros in the spectrum of the graph $G$ is called its nullity.

A recently introduced $[\mathbf{3}, \mathbf{5}]$ spectrum-based graph invariant is

$$
\mathrm{EE}=\mathrm{EE}(G)=\sum_{i=1}^{n} e^{\lambda_{i}}
$$

which we proposed [2] to be called the Estrada index.

The Estrada index has found applications in biochemistry $[\mathbf{3}, \mathbf{4}, \mathbf{7}]$ and in the theory of complex networks $[\mathbf{5}, \mathbf{6}]$. In the paper $[\mathbf{2}]$ lower and upper bounds for EE were deduced. We now obtain some further lower bounds for this graph-spectral invariant.

The eigenvalues of an $(n, m)$-graph satisfy the following elementary conditions $[1]:$

$$
\begin{gathered}
\sum_{i=1}^{n} \lambda_{i}=0 \\
\sum_{i=1}^{n}\left(\lambda_{i}\right)^{2}=2 m .
\end{gathered}
$$

2000 Mathematics Subject Classification: Primary 05C50, 05C35. 
The left-hand sides of equations (1) and (2) are special cases (for $k=0$ and $k=1$ ) of the spectral moments $M_{k}$ defined as

$$
M_{k}=M_{k}(G)=\sum_{i=1}^{n}\left(\lambda_{i}\right)^{k} .
$$

As usual, the complete graph on $n$ vertices will be denoted by $K_{n}$. Its complement, $\bar{K}_{n}$ is the $n$-vertex graph without edges. Its spectrum consists solely of zeros, and therefore $\operatorname{EE}\left(\bar{K}_{n}\right)=n$. In what follows we assume that the graphs considered contain at least one edge $(m>0)$. Such graphs have at least one positive and at least one negative eigenvalue $[\mathbf{1}]$.

At this point we remind the reader that the hyperbolic cosine and hyperbolic sine are defined as

$$
\cosh (x)=\frac{e^{x}+e^{-x}}{2} \quad \text { and } \quad \sinh (x)=\frac{e^{x}-e^{-x}}{2}
$$

respectively.

\section{An auxiliary inequality}

Define two auxiliary quantities $\mathrm{EE}^{-}$and ee as

$$
\begin{aligned}
\mathrm{EE}^{-} & =\mathrm{EE}^{-}(G)=\sum_{i=1}^{n} e^{-\lambda_{i}} \\
\mathrm{ee} & =\operatorname{ee}(G)=\sum_{i=1}^{n} \cosh \left(\lambda_{i}\right) .
\end{aligned}
$$

In view of the power-series expansion of $e^{x}$ and $e^{-x}$, and bearing in mind the definition (3) of spectral moments, we have

$$
\mathrm{EE}=\sum_{k \geqslant 0} \frac{M_{k}}{k !} \quad \text { and } \quad \mathrm{EE}^{-}=\sum_{k \geqslant 0}(-1)^{k} \frac{M_{k}}{k !}
$$

from which

$$
\mathrm{EE}-\mathrm{EE}^{-}=2 \sum_{k \geqslant 0} \frac{M_{2 k+1}}{(2 k+1) !} .
$$

It is known [1] that $M_{k}$ is equal to the number of self-returning walks of length $k$. Consequently, $M_{k} \geqslant 0$ for all graphs and for all $k \geqslant 0$. If the graph $G$ is bipartite, then it contains no odd-membered cycles and, consequently, it has no self-returning walks of odd length, i.e., $M_{k}(G)=0$ for $k=1,3,5,7, \ldots$. In view of this, we conclude that

$$
\operatorname{EE}(G)-\mathrm{EE}^{-}(G) \geqslant 0
$$

and that equality holds if and only if $G$ is bipartite. From (5) follows that

$$
\mathrm{EE}(G) \geqslant \frac{1}{2}\left[\mathrm{EE}(G)+\mathrm{EE}^{-}(G)\right]
$$

i.e.,

$$
\operatorname{EE}(G) \geqslant \operatorname{ee}(G)
$$


Equality in (6) holds if and only if the graph $G$ is bipartite.

In what follows we obtain several lower bounds for ee. Because of (6) these will also be lower bounds for EE.

\section{An $(n, m)$-type lower bound}

A vertex of a graph, having degree zero is said to be isolated.

THEOREM 1. If $G$ is an $(n, m)$-graph without isolated vertices, then

$$
\operatorname{EE}(G) \geqslant n \cosh \left(\sqrt{\frac{2 m}{n}}\right) .
$$

Equality holds if and only if $G$ is regular of degree 1.

Proof. Because of (6), in order to obtain the inequality (7) it is sufficient to demonstrate the validity of

$$
\mathrm{ee}(G) \geqslant n \cosh \left(\sqrt{\frac{2 m}{n}}\right) .
$$

First of all, note that $2 m / n$ is the average vertex degree of the respective graph $G$. Therefore, if $G$ has no isolated vertices, then $2 m / n \geqslant 1$.

We now use a Lagrange-multiplier technique to arrive at an extremal value of $\operatorname{ee}(G)$. That this is a minimum will be verified at a later moment.

Consider thus the expression

$$
F=\mathrm{ee}-\frac{1}{2} \alpha\left(\sum_{i=1}^{m}\left(\lambda_{i}\right)^{2}-2 m\right)
$$

by means of which we seek for an extremal value of ee under the condition that relation (2) is obeyed. The equations that need to be satisfied are:

$$
\frac{\partial F}{\partial \lambda_{k}}=0 \quad \text { for } k=1,2, \ldots, n
$$

i.e.,

$$
\sinh \left(\lambda_{k}\right)-\alpha \lambda_{k}=0 \quad \text { for } k=1,2, \ldots, n .
$$

It is easy to see that if $\alpha>1$, then the equation

$$
\sinh (x)-\alpha x=0
$$

has three solutions: $x_{0}>0,0$, and $-x_{0}$. Thus, in order to obtain an extremal value $\mathrm{ee}^{*}$ of ee we have to substitute into the right-hand side of (4) the numbers $x_{0}, 0$, and $-x_{0}$ instead of the eigenvalues $\lambda_{i}$. Let the number of eigenvalues that are replaced by 0 be $p$. Then the remaining $n-p$ eigenvalues are replaced by $x_{0}$ and/or $-x_{0}$, resulting in

$$
\mathrm{ee}^{*}=p+(n-p) \cosh \left(x_{0}\right) .
$$


In order that relation (2) be obeyed, it must be $(n-p)\left(x_{0}\right)^{2}=2 m$, from which $x_{0}=\sqrt{2 m /(n-p)}$ and

$$
\mathrm{ee}^{*}=p+(n-p) \cosh \left(\sqrt{\frac{2 m}{n-p}}\right) .
$$

Because $2 m / n \geqslant 1$, for any value of $p$ it will be $x_{0} \geqslant 1$ and therefore $\cosh \left(x_{0}\right) \geqslant$ $\cosh (1)=1.543080 \ldots$. Thus the right-hand side of (11) may be viewed as consisting of $p$ summands equal to 1 , and $n-p$ summands greater than 1.54. Evidently, this expression will attain its greatest possible value for $p=0$.

Therefore, if ee* is a lower bound for ee (which still needs to be verified), then the best such lower bound is for $p=0$, which is just the right-hand side expression in (7).

What remains to be proven is that the Lagrange multiplier $\alpha$ is indeed greater than unity, and that $\mathrm{ee}^{*}$ for $p=0$ is a minimum.

From (10) we obtain that

$$
\alpha=\frac{\sinh \left(x_{0}\right)}{x_{0}}
$$

and we recall that $x_{0} \geqslant 1$. Because

$$
\left(\frac{\sinh (x)}{x}\right)^{\prime}=\frac{(x-1) e^{x}+(x+1) e^{-x}}{2 x}
$$

it is evident that for $x \geqslant 1$ the function $\sinh (x) / x$ is monotonically increasing. Since for $x=1, \sinh (x) / x=1.175201 \ldots$, it must be $\alpha>1$.

In order to show that the right-hand side of Eq. (8) is a minimum, we have to examine the Hessian matrix of the function $F$, Eq. (9). Direct calculation yields:

$$
\begin{aligned}
\frac{\partial^{2} F}{\partial \lambda_{k}^{2}} & =\cosh \left(\lambda_{k}\right)-\alpha \\
\frac{\partial^{2} F}{\partial \lambda_{k} \partial \lambda_{k^{\prime}}} & =0 \quad \text { for } \quad k \neq k^{\prime} .
\end{aligned}
$$

Thus, the Hessian matrix is diagonal and all its diagonal elements are equal to

$$
\cosh \left(\lambda_{k}\right)-\alpha=\cosh \left(x_{0}\right)-\frac{\sinh \left(x_{0}\right)}{x_{0}}=\frac{\left(x_{0}-1\right) e^{x_{0}}+\left(x_{0}+1\right) e^{-x_{0}}}{2 x_{0}}
$$

which for $x_{0} \geqslant 1$ is evidently positive-valued. Therefore ee* is a minimum, and inequality (8) is obeyed. Then also inequality (7) holds.

Equality in (7) will be attained if the underlying graph is bipartite and if all its eigenvalues are equal by absolute value. This latter condition is satisfied only the regular graph of degree one, i. e., by the graph consisting of $n / 2$ copies of $K_{2}$. For this graph $2 m / n=1$ and therefore $\mathrm{EE}=n \cosh (1)$.

This completes the proof of Theorem 1 .

REMARK 2. It is interesting to note that in the proof of Theorem 1, the condition (1) has not been taken into account. Taking into account condition (1), or any other condition that the graph eigenvalues satisfy, was possible, but not necessary. 
It was our (successful) choice to pursue a Lagrange-multiplier approach based solely on condition (2). If condition (1) would be included into the test-function (9), a better bound for EE could be expected, but then a much more complicated situation would occur: the equation analogous to (10) would have either two positive and one negative solution, or one positive and two negative solutions. Finding the respective expression for ee* would become a difficult and infeasible task.

Theorem 1 can be somewhat strengthened. Namely, in its proof not the absence of isolated vertices, but the condition $2 m / n \geqslant 1$ was used. In view of this we have:

Theorem 3. If $G$ is an $(n, m)$-graph for which $2 m / n \geqslant 1$, then

$$
\operatorname{EE}(G) \geqslant n \cosh \left(\sqrt{\frac{2 m}{n}}\right) .
$$

Equality holds if and only if $G$ is regular of degree 1.

If the number of isolated vertices in the $(n, m)$-graph $G$ is known, and is, say, equal to $q$, then $G=G^{*} \cup \overline{K_{q}}$, where $G^{*}$ is an $(n-q, m)$-graph to which Theorem 1 is applicable. Because $\operatorname{EE}(G)=q+\operatorname{EE}\left(G^{*}\right)$, we immediately arrive at:

Corollary 4. If $G$ is an $(n, m)$-graph with exactly $q$ isolated vertices $(q<n)$, then

$$
\mathrm{EE}(G) \geqslant q+(n-q) \cosh \left(\sqrt{\frac{2 m}{n-q}}\right) .
$$

Equality holds if and only if $G$ consists of a regular graph of degree 1 and $q$ isolated vertices.

\section{The case $2 m / n<1$}

If the average vertex degree $2 m / n$ is less than unity, then the graph $G$ necessarily possesses isolated vertices. If the number of isolated vertices is known, then Corollary 3 is applicable. Otherwise we may proceed as follows.

If $2 m / n<1$, then the number of isolated vertices in $G$ is at least $n-2 m$, so that $G$ can be written as $G=G^{\dagger} \cup \bar{K}_{n-2 m}$. The graph $G^{\dagger}$ may still possess isolated vertices. However, $G^{\dagger}$ possesses exactly $2 m$ vertices, and therefore its average vertex degree is unity. Consequently, by Theorem 2 ,

$$
\mathrm{EE}\left(G^{\dagger}\right) \geqslant 2 m \cosh (1)
$$

and we arrive at:

TheOREM 5. If $G$ is an $(n, m)$-graph for which $2 m / n<1$, then

$$
\operatorname{EE}(G) \geqslant n-2 m+2 m \cosh (1) .
$$

Equality is attained if and only if $G$ consists of $n-2 m$ isolated vertices and a regular graph of degree 1 on $2 m$ vertices. 


\section{An $\left(n, m, n_{0}\right)$-type lower bound}

Suppose that in addition to the parameters $n$ and $m$ we know also the nullity $n_{0}$ of the graph $G$. If $n_{0}=0$, then $G$ does not possess isolated vertices, and Theorem 1 is applicable. We therefore assume that $n_{0}>0$. Then we have:

TheOREM 6. If $G$ is an $(n, m)$-graph with at least one edge and nullity $n_{0}>0$, then

$$
\mathrm{EE}(G) \geqslant n_{0}+\left(n-n_{0}\right) \cosh \left(\sqrt{\frac{2 m}{n-n_{0}}}\right) .
$$

Equality holds if and only if $G$ consists either of isolated vertices and copies of $K_{2}$, or of isolated vertices and copies of various complete bipartite graphs $K_{a, b}$, such that the product $a \cdot b$ is constant.

REMARK 7. An example of a graph for which equality in (12) holds is the graph consisting of a copy of $K_{1,12}$, two copies of $K_{2,6}$, three copies of $K_{3,4}$, and four isolated vertices. Its spectrum consists of the numbers $\sqrt{12}$ (with multiplicity 6 ), 0 (with multiplicity 42 ), and $-\sqrt{12}$ (with multiplicity 6 ). Therefore

$$
\mathrm{EE}=42+6 e^{\sqrt{12}}+6^{-\sqrt{12}}
$$

which coincides with the right-hand side of (12) for $n=54, m=72$, and $n_{0}=42$.

Proof. The proof of Theorem 5 is analogous to the proof of Theorem 1 and we point out only the main differences. Label the eigenvalues of $G$ so that $\lambda_{i}=0$ for $n-n_{0}+1 \leqslant i \leqslant n$. Then

$$
\operatorname{EE}(G) \leqslant n_{0}+\mathrm{ee}_{0}(G)
$$

and the auxiliary quantity to be minimized is

$$
\mathrm{ee}_{0}(G)=\sum_{i=1}^{n-n_{0}} \cosh \left(\lambda_{i}\right)
$$

At a pertinent point of the proof one needs to show that for any graph $G$ with at least one edge, $2 m /\left(n-n_{0}\right) \geqslant 1$. To see this, suppose that the graph $G$ has exactly $q$ isolated vertices. Then $G=G^{*} \cup \bar{K}_{q}$ and

$$
\begin{aligned}
m(G) & =m\left(G^{*}\right) \\
n(G) & =n\left(G^{*}\right)+q \\
n_{0}(G) & =n_{0}\left(G^{*}\right)+q .
\end{aligned}
$$

Bearing in mind that $G^{*}$ possesses at least one edge and therefore at least one positive eigenvalue, we have

$$
\frac{2 m(G)}{n(G)-n_{0}(G)}=\frac{2 m\left(G^{*}\right)}{n\left(G^{*}\right)-n_{0}\left(G^{*}\right)}>\frac{2 m\left(G^{*}\right)}{n\left(G^{*}\right)} \geqslant 1 .
$$

The rest of the proof is same as in Theorem 1 . 


\section{References}

[1] D. Cvetković, M. Doob, H. Sachs, Spectra of Graphs - Theory and Application, third ed., Johann Ambrosius Barth Verlag, Heidelberg, 1995.

[2] J.A. de la Peña, I. Gutman, J. Rada, Estimating the Estrada index, Lin. Algebra Appl. 56 (2008), 507-509.

[3] E. Estrada, Characterization of 3D molecular structure, Chem. Phys. Lett. 319 (2000), 713718.

[4] E. Estrada, Characterization of the folding degree of proteins, Bioinformatics 18 (2002), 697704.

[5] E. Estrada, J. A. Rodríguez-Velázquez, Subgraph centrality in complex networks, Phys. Rev. E71 (2005), 056103-1-9.

[6] E. Estrada, J. A. Rodríguez-Velázquez, Spectral measures of bipartivity in complex networks, Phys. Rev. E72 (2005), 046105-1-6.

[7] E. Estrada, J. A. Rodríguez-Velázquez, M. Randić, Atomic branching in molecules, Int. J. Quantum Chem. 106 (2006), 823-832.

Faculty of Science

(Received 1101 2007)

University of Kragujevac

P.O. Box 60

34000 Kragujevac

Serbia

gutman@kg.ac.yu 